\title{
A New Generation of Glycoconjugated Azo Dyes Based on Aminosugars
}

\author{
Lorenzo Guazzelli, ${ }^{1,2}$ Giorgio Catelani, ${ }^{1}$ and Felicia D'Andrea ${ }^{1}$ \\ ${ }^{1}$ Department of Pharmacy, University of Pisa, Via Bonanno 33, 56126 Pisa, Italy \\ ${ }^{2}$ School of Chemistry and Chemical Biology, University College Dublin, Belfield, Dublin 4, Ireland \\ Correspondence should be addressed to Lorenzo Guazzelli; lorenzo.guazzelli@ucd.ie \\ and Giorgio Catelani; giorgio.catelani@farm.unipi.it
}

Received 7 May 2015; Revised 13 July 2015; Accepted 14 July 2015

Academic Editor: Vladimir Kren

Copyright (C) 2015 Lorenzo Guazzelli et al. This is an open access article distributed under the Creative Commons Attribution License, which permits unrestricted use, distribution, and reproduction in any medium, provided the original work is properly cited.

The third generation of glycoconjugated azo dyes (GADs) was prepared linking monoazo dyes to 6-amino-6-deoxy-D-galactose or $6^{\prime}$ amino- $6^{\prime}$-deoxylactose through mixed amido-ester connections. The complementary conjugation reactions were studied using the succinyl derivative of either the acetal protected aminosugar or the azo dye. Target "naturalized" GADs were obtained after acid hydrolysis of the acetal protecting groups present on the sugar moiety.

\section{Introduction}

The most commonly employed class of industrial textile dyes are the so-called "disperse dyes" family, characterised by their extremely low solubility in water. These, in a finely dispersed state, are used in dyeing processes of synthetic fibres, such as polyester, polyacetate, and polyamide. Owing to the absence of ionic groups in these synthetic fibres, only apolar dyes can be utilized [1-3]. Among the various troublesome aspects related to the use of disperse dyes and their dyeing processes, the highest concerns are raised by (a) the use of substantial amounts of dispersing agents, which are needed to bring the insoluble dyes in a stable colloidal dispersion throughout the application process, and (b) the use of high temperatures, typically around $130^{\circ} \mathrm{C}$, which demand large amounts of energy and require appropriate dyeing machines able to operate under pressure [1-3]. Azo and anthraquinone dyes represent the two most relevant types of disperse dyes. Although disperse dyes can differ considerably in molecular weight, only those with a low mass (typically between 200 and $500 \mathrm{Da}$ ) [1] are effectively used in the dyeing of textile apolar synthetic fibres.

Recently, an innovative class of azo dyes [4-6] has been proposed. These have been obtained through the glycoconjugation of common mono- and disaccharides, such as D- glucose, D-galactose, and lactose, with some model synthetic monoazo dyes. Glycoconjugated azo dyes (GADs) have been prepared by the so-called "naturalisation" procedure, which is to link the azo dye component and the sugar component through difunctional spacers by means of either two consecutive esterification reactions or two consecutive alkylation reactions. Glycoconjugated azo dyes (GADs, Figure 1) type $1[4,5]$ and type $2[6]$ characterised, respectively, by diester and diether bonds have been evaluated for their tinctorial properties, giving unexpected results. In fact, GADs dye synthetic fibres quickly in boiling water, at ambient pressure, and without the need of dispersant, thanks to the increased hydrophilic characteristics. Uniquely, GADs can also be used effectively to dye natural fibres, such as wool and silk, allowing the study of solutions to novel dyeing technologies. Moreover, biodegradation and detoxification of a selected diestereal GAD by Fusarium oxysporum gave promising results [7] for the treatment of the aqueous waste, representing a step further towards environmentally friendly tinctorial processes.

In a more recent investigation [8] high molecular weight anthraquinone or bis-azo disperse dyes only become watersoluble after conjugation with two lactose units. The doubleconjugation procedure required a preliminary insertion of a malonate spacer followed by condensation with two $6^{\prime}$ - 


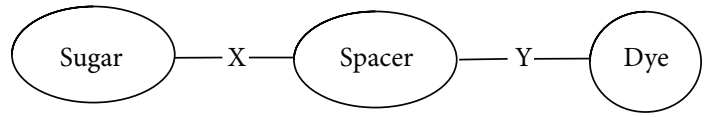

(1) $\mathrm{X}=\mathrm{Y}=\mathrm{OCO}$

(2) $\mathrm{X}=\mathrm{Y}=\mathrm{O}$

(3) $\mathrm{X}=\mathrm{NHCO}, \mathrm{Y}=\mathrm{OCO}$

FIGURE 1: General structure of glycoconjugated azo dyes (GADs).

amino- $6^{\prime}$-deoxylactose units [8]. Also, glutamic acid was used as the additional spacer in order to allow the doubleconjugation [9]. Presented here is an extension of our previous results, namely, the synthesis of the third generation of naturalized GADs by conjugation of low molecular weight monoazo dyes with either 6-amino-6-deoxy-D-galactose or $6^{\prime}$-amino- $6^{\prime}$-deoxylactose through mixed amido-ester connections (3).

\section{Experimental}

Optical rotations were measured with a Perkin-Elmer 241 polarimeter at the sodium $\mathrm{D}$-line $(589 \mathrm{~nm})$ at $20 \pm 2^{\circ} \mathrm{C}$ using a $1 \mathrm{dm}$ cell. ${ }^{1} \mathrm{H}$ NMR and ${ }^{13} \mathrm{C}$ NMR spectra were recorded with a Bruker AC 200 operating at $200.13\left({ }^{1} \mathrm{H}\right)$ and $50.3 \mathrm{MHz}\left({ }^{13} \mathrm{C}\right)$ or with a Bruker Avance II 250 spectrometer operating at $250.12\left({ }^{1} \mathrm{H}\right)$ and $62.9 \mathrm{MHz}\left({ }^{13} \mathrm{C}\right)$. Spin resonances were reported as chemical shifts $(\delta)$ in part per million (ppm) and the references to the residual peak of the solvent employed as follows: $\mathrm{CDCl}_{3} 7.27 \mathrm{ppm}\left({ }^{1} \mathrm{H} \mathrm{NMR}\right)$ and $77.0 \mathrm{ppm}\left({ }^{13} \mathrm{C} \mathrm{NMR}\right.$, central band), $\mathrm{CD}_{3} \mathrm{CN} 1.94 \mathrm{ppm}\left({ }^{1} \mathrm{H}\right.$ NMR, central band) and $1.28 \mathrm{ppm}\left({ }^{13} \mathrm{C}\right.$ NMR, central band), and $\mathrm{CD}_{3} \mathrm{OD} 3.31 \mathrm{ppm}$ $\left({ }^{1} \mathrm{H}\right.$ NMR, central band) and $49.0 \mathrm{ppm}\left({ }^{13} \mathrm{C}\right.$ NMR, central band). Coupling constants $J$ were reported in Hertz $(\mathrm{Hz})$. The assignments were made, when possible, with the aid of DEPT, HETCOR, COSY, and HSQC experiments and in the case of anomeric mixtures, referring to the differences in the peak intensities and comparison with values for known analogous compounds. UV-Vis spectra were recorded on a Cary-300 Agilent Technologies Cary Series UV-Vis Spectrophotometer. Elemental analyses were performed by Carlo Erba elemental analyzer MOD 1106 instrument. All reactions were followed by TLC on Kieselgel $60 \mathrm{~F}_{254}$ with detection by UV light and/or with ethanolic $10 \%$ phosphomolybdic or sulfuric acid and heating. Kieselgel 60 (E. Merck, 70-230 and 230-400 mesh, resp.) was used for column and flash chromatography. Solvents were dried and purified by distillation according to standard procedure [10] and stored over $4 \AA$ molecular sieves activated for at least $24 \mathrm{~h}$ at $200^{\circ} \mathrm{C}$. $\mathrm{Na}_{2} \mathrm{SO}_{4}$ or $\mathrm{MgSO}_{4}$ was used as the drying agent for solutions. 6-Amino-6-deoxy-1,2:3,4-di-Oisopropylidene- $\alpha$-D-galactopyranose (4) [11], 6-amino-6deoxy-3,4- $O$-isopropylidene- $\beta$-D-galactopyranosyl- $(1 \rightarrow 4)$ 2,3:5,6-di-O-isopropylidene-aldehydo-D-glucose dimethyl acetal (5) [8], 2-[ethyl-(4-phenyldiazenyl)-phenyl-amino]ethanol (8) [12], and 4-[2-ethyl-(\{4-[(4-nitrophenyl)dia- zenyl]phenyl\}amino)ethoxy]-4-oxobutanoic acid (10) [4] were prepared through literature methods.

2.1. 6-N-(3-Carboxy-propanoyl)-6-deoxy-1,2:3,4-di-O-isopropylidene- $\alpha$-D-galactopyranose (6). A solution of 4 [11] $(2.60 \mathrm{~g}, 10.0 \mathrm{mmol})$ in $\mathrm{MeOH}(70 \mathrm{~mL})$ was treated with succinic anhydride $(1.20 \mathrm{~g}, 12.0 \mathrm{mmol}, 1.2 \mathrm{eq})$ and stirred at room temperature until the starting material was completely disappeared (TLC, 9:1 EtOAc- $\left.{ }^{\mathrm{i}} \mathrm{PrOH}\right)$. After $2 \mathrm{~h}, \mathrm{Et}_{3} \mathrm{~N}(3 \mathrm{~mL})$ was added to the reaction mixture, which was stirred for $10 \mathrm{~min}$ at room temperature and then was repeatedly coevaporated with toluene $(5 \times 20 \mathrm{~mL})$ at diminished pressure. The resulting residue was dissolved into $\mathrm{CH}_{2} \mathrm{Cl}_{2}$ $(50 \mathrm{~mL})$, neutralized with $\mathrm{AcOH}(6 \mathrm{~mL})$, and washed with water $(25 \mathrm{~mL})$ and the aqueous phase was extracted with $\mathrm{CH}_{2} \mathrm{Cl}_{2}(4 \times 30 \mathrm{~mL})$. The combined organic layers were dried $\left(\mathrm{MgSO}_{4}\right)$, filtered, and concentrated under diminished pressure. The crude residue $(3.42 \mathrm{~g})$ was subjected to flash chromatographic purification on silica gel (EtOAc) to give pure 6 as white foam $(2.75 \mathrm{~g}, 76 \%$ yield $) ;[\alpha]_{\mathrm{D}}-9.8$ (c 1.1, $\left.\mathrm{CHCl}_{3}\right) ; R_{f} 0.50$ (9:1 EtOAc- $\left.{ }^{\mathrm{i}} \mathrm{PrOH}\right) ;{ }^{1} \mathrm{H}$ NMR $(200.13 \mathrm{MHz}$, $\left.\mathrm{CDCl}_{3}\right): \delta 1.29,1.31,1.38,1.43\left[4 \mathrm{~s}\right.$, each $3 \mathrm{H}, 2 \times \mathrm{C}\left(\mathrm{CH}_{3}\right)_{3}$ ]; $2.41-2.55\left[\mathrm{~m}, 4 \mathrm{H},\left(\mathrm{CH}_{2}\right)_{2}\right.$ ], $3.16\left(\mathrm{ddd}, 1 \mathrm{H}, J_{6 \mathrm{a}, 6 \mathrm{~b}} 13.7 \mathrm{~Hz}, J_{6 \mathrm{a}, \mathrm{NH}}\right.$ $\left.5.3 \mathrm{~Hz}, J_{5,6 \mathrm{a}} 8.0 \mathrm{~Hz}, \mathrm{H}-6 \mathrm{a}\right), 3.42$ (ddd, $1 \mathrm{H}, J_{6 \mathrm{~b}, \mathrm{NH}} 6.4 \mathrm{~Hz}$, $J_{5,6 \mathrm{~b}} 4.7 \mathrm{~Hz}, \mathrm{H}-6 \mathrm{~b}$ ), 3.86 (ddd, $\left.1 \mathrm{H}, \mathrm{H}-5\right), 4.19$ (dd, $1 \mathrm{H}, J_{3,4}$ $\left.7.9 \mathrm{~Hz}, J_{4,5} 1.8 \mathrm{~Hz}, \mathrm{H}-4\right), 4.31\left(\mathrm{dd}, 1 \mathrm{H}, J_{1,2} 5.0 \mathrm{~Hz}, J_{2,3} 2.4 \mathrm{~Hz}\right.$, H-2), 4.59 (dd, $1 \mathrm{H}, \mathrm{H}-3$ ), 5.43 (d, $1 \mathrm{H}, \mathrm{H}-1), 6.80$ (bt, $1 \mathrm{H}$, $\mathrm{NH}) ;{ }^{13} \mathrm{C}$ NMR $\left(50.3 \mathrm{MHz}, \mathrm{CDCl}_{3}\right): \delta 24.6,25.2,26.2,26.3$ $\left[2 \times \mathrm{C}\left(\mathrm{CH}_{3}\right)_{3}\right] ; 30.2,31.0\left[\left(\mathrm{CH}_{2}\right)_{2}\right], 40.8(\mathrm{C}-6), 66.9(\mathrm{C}-5)$, 71.4, 71.6, 72.2, (C-2, C-3, C-4), 97.1 (C-1), 109.4, 109.8 [2 $\times$ $\left.\mathrm{C}\left(\mathrm{CH}_{3}\right)_{3}\right], 173.8,174.6(2 \times \mathrm{C}=\mathrm{O})$, Anal. Calcd for $\mathrm{C}_{16} \mathrm{H}_{25} \mathrm{NO}_{8}$ (359.37): C, 53.47; H, 7.01; N, 3.90. Found: C, 53.44; H, 6.99; $\mathrm{N}, 3.88$.

2.2. 6-N-(3-Carboxy-propanoyl)-6-deoxy-3,4-O-isopropylidene- $\beta$-D-galactopyranosyl-(1 $\rightarrow 4)-2,3: 5,6-d i$-O-isopropylidene-aldehydo-D-glucose Dimethyl Acetal (7). A solution of amine 5 [8] (1.00 g, $1.97 \mathrm{mmol})$ and succinic anhydride (216 mg, $2.16 \mathrm{mmol}, 1.2 \mathrm{eq})$ in $\mathrm{MeOH}(40 \mathrm{~mL})$ was allowed to react in the reaction conditions described for the preparation of $\mathbf{6}$, leading to the residue $(1.17 \mathrm{~g})$ constituted exclusively $\left({ }^{13} \mathrm{C}\right.$ NMR) by 7 (98\% yield). An analytical sample of 7 was obtained through flash chromatography over silica gel eluting with 9:1 EtOAc ${ }^{\mathrm{i}} \mathrm{PrOH}$. Compound 7 was a white foam; $[\alpha]_{\mathrm{D}}+53.2\left(\right.$ c 1.1, $\left.\mathrm{CHCl}_{3}\right) ; R_{f}$ 0.51 (9:1 EtOAc- $\left.{ }^{\mathrm{i}} \mathrm{PrOH}\right)$; ${ }^{1} \mathrm{H}$ NMR $\left(250.12 \mathrm{MHz}, \mathrm{CD}_{3} \mathrm{CN}\right): \delta 1.29,1.34[2 \mathrm{~s}$, each $6 \mathrm{H}$, $\left.2 \times \mathrm{C}\left(\mathrm{CH}_{3}\right)_{3}\right], 1.39,1.43\left[2 \mathrm{~s}\right.$, each $\left.3 \mathrm{H}, \mathrm{C}\left(\mathrm{CH}_{3}\right)_{3}\right], 2.51[\mathrm{~m}$, $\left.4 \mathrm{H},\left(\mathrm{CH}_{2}\right)_{2}\right], 3.44,3.49\left(2 \mathrm{~s}\right.$, each $\left.3 \mathrm{H}, 2 \times \mathrm{OCH}_{3}\right), 3.13$ (ddd, $\left.1 \mathrm{H}, J_{5^{\prime}, 6^{\prime} \mathrm{a}} 6.8 \mathrm{~Hz}, J_{6^{\prime} \mathrm{a}, \mathrm{NH}} 4.9 \mathrm{~Hz}, J_{6^{\prime} \mathrm{a}, 6^{\prime} \mathrm{b}} 13.8 \mathrm{~Hz}, \mathrm{H}-6^{\prime} \mathrm{a}\right), 3.33$ $\left(\mathrm{dd}, 1 \mathrm{H}, J_{1^{\prime}, 2^{\prime}} 8.1 \mathrm{~Hz}, J_{2^{\prime}, 3^{\prime}} 7.3 \mathrm{~Hz}, \mathrm{H}-2^{\prime}\right), 3.67-3.80(\mathrm{~m}, 2 \mathrm{H}$, H-5', H-6'b), 3.84 (dd, $1 \mathrm{H}, J_{3,4} 1.3 \mathrm{~Hz}, J_{4,5} 5.4 \mathrm{~Hz}, \mathrm{H}-4$ ), $3.99\left(\mathrm{dd}, 1 \mathrm{H}, J_{3^{\prime}, 4^{\prime}} 3.2 \mathrm{~Hz}, \mathrm{H}-3^{\prime}\right), 4.06(\mathrm{~m}, 3 \mathrm{H}, \mathrm{H}-3, \mathrm{H}-6 \mathrm{a}$, $\mathrm{OH}-2^{\prime}$ ), 4.09 (dd, $\left.1 \mathrm{H}, J_{4^{\prime}, 5^{\prime}} 5.1 \mathrm{~Hz}, \mathrm{H}-4^{\prime}\right), 4.22$ (m, 2H, H-5, H-6b), $4.33\left(\mathrm{~d}, 1 \mathrm{H}, \mathrm{H}-\mathrm{1}^{\prime}\right), 4.36\left(\mathrm{~d}, 1 \mathrm{H}, J_{1,2} 6.4 \mathrm{~Hz}, \mathrm{H}-1\right)$, $4.41\left(\mathrm{dd}, 1 \mathrm{H}, J_{2,3} 7.1 \mathrm{~Hz}, \mathrm{H}-2\right), 6.85$ (bt, $\left.1 \mathrm{H}, \mathrm{NH}\right) ;{ }^{13} \mathrm{C}$ NMR $\left(62.9 \mathrm{MHz}, \mathrm{CD}_{3} \mathrm{CN}\right): \delta 25.2,26.5,26.6,26.9,27.4,28.4[3 \times$ $\left.\mathrm{C}\left(\mathrm{CH}_{3}\right)_{3}\right], 30.1,31.0\left[\left(\mathrm{CH}_{2}\right)_{2}\right], 58.1,56.0\left(2 \times \mathrm{OCH}_{3}\right), 41.1$ 
$\left(\mathrm{C}-6^{\prime}\right), 66.1(\mathrm{C}-6), 74.6\left(\mathrm{C}-2^{\prime}\right), 72.5\left({\mathrm{C}-5^{\prime}}^{\prime}\right), 75.0\left(\mathrm{C}-4^{\prime}\right), 77.4$ (C-2), 77.6 (C-5), 77.7 (C-4), 78.4 (C-3), 80.0 (C-3'), 104.0 $\left(\mathrm{C}-1^{\prime}\right), 108.2(\mathrm{C}-1), 109.3,110.4,110.7\left[3 \times \mathrm{C}\left(\mathrm{CH}_{3}\right)_{3}\right], 173.6$, $174.7(2 \times \mathrm{C}=\mathrm{O})$. Anal. Calcd for $\mathrm{C}_{27} \mathrm{H}_{45} \mathrm{NO}_{14}$ (607.28): C, 53.37; H, 7.46; N, 2.31. Found: C, 53.35; H, 7.43; N, 2.29.

2.3. Preparation of Protected GAD 11. A solution of 6 $(1.50 \mathrm{~g}, 4.17 \mathrm{mmol})$ and azo dye $9(1.81 \mathrm{~g}, 5.17 \mathrm{mmol}$, $1.24 \mathrm{eq})$ in dry $\operatorname{THF}(50 \mathrm{~mL})$ was treated with $N, N^{\prime}$ dicyclohexylcarbodiimide (DDC) $(1.03 \mathrm{~g}, 5.0 \mathrm{mmol}, 1.2 \mathrm{eq})$ and 4-dimethylaminopyridine (DMAP) (145 mg, $1.19 \mathrm{mmol})$ and the solution was stirred at room temperature until the starting material was completely disappeared (4h, TLC, EtOAc). The reaction mixture was concentrated under diminished pressure and the crude residue was dissolved into $\mathrm{CH}_{2} \mathrm{Cl}_{2}(70 \mathrm{~mL})$ and washed in the order with saturated aqueous $\mathrm{NaHCO}_{3}(3 \times 30 \mathrm{~mL})$ and brine $(30 \mathrm{~mL})$. The organic phase was dried $\left(\mathrm{Na}_{2} \mathrm{SO}_{4}\right)$, filtered, and concentrated under diminished pressure. Purification of the crude residue $(3.10 \mathrm{~g})$ by flash chromatography over silica gel (2:3 hexaneEtOAc) gave pure $\mathbf{1 1}$ as a red foam $\left(2.14 \mathrm{~g}, 74 \%\right.$ yield); $R_{f}$ 0.22 (2:3 hexane-EtOAc); ${ }^{1} \mathrm{H}$ NMR (200.13 MHz, $\left.\mathrm{CDCl}_{3}\right): \delta$ $1.27\left(\mathrm{t}, 3 \mathrm{H}, J 7.2 \mathrm{~Hz}, \mathrm{CH}_{3}\right), 1.32,1.33,1.45,1.48$, [4s, each $3 \mathrm{H}$, $\left.2 \times \mathrm{C}\left(\mathrm{CH}_{3}\right)_{3}\right], 2.65,2.48\left[2 \mathrm{~m}\right.$, each $\left.2 \mathrm{H},\left(\mathrm{CH}_{2}\right)_{2}\right], 3.20(\mathrm{ddd}$, $\left.1 \mathrm{H}, J_{6 \mathrm{a}, 6 \mathrm{~b}} 13.9 \mathrm{~Hz}, J_{5,6 \mathrm{a}} 8.9 \mathrm{~Hz}, J_{6 \mathrm{a}, \mathrm{NH}} 3.7 \mathrm{~Hz}, \mathrm{H}-6 \mathrm{a}\right), 3.55$ (q, $\left.2 \mathrm{H}, J 7.2 \mathrm{~Hz}, \mathrm{CH}_{3} \mathrm{CH}_{2} \mathrm{~N}\right), 3.70\left(\mathrm{t}, 2 \mathrm{H}, J 6.2 \mathrm{~Hz}, \mathrm{OCH}_{2} \mathrm{CH}_{2} \mathrm{~N}\right)$, 3.72 (ddd, $1 \mathrm{H}, J_{5,6 \mathrm{~b}} 3.5 \mathrm{~Hz}, J_{6 \mathrm{~b}, \mathrm{NH}} 7.2 \mathrm{~Hz}, \mathrm{H}-6 \mathrm{~b}$ ), 3.87 (ddd, $1 \mathrm{H}, \mathrm{H}-5), 4.19$ (dd, $\left.1 \mathrm{H}, J_{4,5} 1.7 \mathrm{~Hz}, J_{3,4} 7.9 \mathrm{~Hz}, \mathrm{H}-4\right), 4.31$ (dd, $\left.1 \mathrm{H}, J_{1,2} 5.0 \mathrm{~Hz}, J_{2,3} 2.5 \mathrm{~Hz}, \mathrm{H}-2\right), 4.32$ (t, $2 \mathrm{H}, J 6.2 \mathrm{~Hz}$, $\mathrm{CH}_{2} \mathrm{OCO}$ ), 4.59 (dd, 1H, H-3), 5.51 (d, 1H, H-1), 5.98 (dd, $1 \mathrm{H}, \mathrm{NH}), 7.79$ (d, 1H, Ar-H-6), 6.87, $7.96\left(\mathrm{AA}^{\prime} \mathrm{XX}^{\prime}\right.$ system, 4H, Ar-H-8, Ar-H-9, Ar-H-11, Ar-H-12), 8.16 (dd, 1H, J5,6 $9.0 \mathrm{~Hz}, \mathrm{Ar}-\mathrm{H}-5), 8.40$ (d, $\left.1 \mathrm{H}, J_{3,5} 2.4 \mathrm{~Hz}, \mathrm{Ar}-\mathrm{H}-3\right) ;{ }^{13} \mathrm{C} \mathrm{NMR}$ $\left(50.3 \mathrm{MHz}, \mathrm{CDCl}_{3}\right): \delta 12.2\left(\mathrm{CH}_{3}\right), 24.3,24.9,25.9,26.0[2 \times$ $\left.\mathrm{C}\left(\mathrm{CH}_{3}\right)_{3}\right], 29.3,30.6\left[\left(\mathrm{CH}_{2}\right)_{2}\right], 40.0(\mathrm{C}-6), 45.8\left(\mathrm{CH}_{3} \mathrm{CH}_{2} \mathrm{~N}\right)$, $48.8\left(\mathrm{OCH}_{2} \mathrm{CH}_{2} \mathrm{~N}\right), 61.3\left(\mathrm{CH}_{2} \mathrm{OCO}\right), 66.3(\mathrm{C}-5), 70.5(\mathrm{C}-2)$, 70.6 (C-3), 71.6 (C-4), 96.1 (C-1), 108.7, $109.4\left[2 \times \mathrm{C}\left(\mathrm{CH}_{3}\right)_{3}\right]$, $111.5,111.6,117.9,122.5,125.9,126.9,126.9(7 \times \mathrm{Ar}-\mathrm{CH})$, 133.9 (Ar-C-2), 144.4 (Ar-C-7), 147.1 (Ar-C-4), 151.6, 153.0 (Ar-C-1, Ar-C-10), 171.3, $172.7(2 \times \mathrm{C}=\mathrm{O})$. Anal. Calcd for $\mathrm{C}_{32} \mathrm{H}_{40} \mathrm{ClN}_{5} \mathrm{O}_{10}$ (690.14): C, 55.69; H, 5.84; N, 10.15. Found: C, 55.66; H, 5.83; N, 10.12 .

2.4. Preparation of Protected GAD 12. The condensation of $7(1.50 \mathrm{~g}, 2.47 \mathrm{mmol})$ and azo dye 9 (1.06 g, $3.05 \mathrm{mmol}$, $1.24 \mathrm{eq})$ with $N, N^{\prime}$-dicyclohexylcarbodiimide (DDC) (630 $\mathrm{mg}, 3.05 \mathrm{mmol}$ ) and 4-dimethylaminopyridine (DMAP) $(90 \mathrm{mg}, 0.73 \mathrm{mmol})$ in dry THF $(30 \mathrm{~mL})$ was performed according to the procedure described above for $\mathbf{1 1}$. The flash chromatography over silica gel of the crude product (1:4 hexane-EtOAc) led to pure $12(1.90 \mathrm{~g}, 82 \%$ yield) as a red foam; $R_{f} 0.27$ (1:4 hexane-EtOAc); $\lambda_{\max } 493.00 \mathrm{~nm}(\mathrm{MeOH})$; ${ }^{1} \mathrm{H}$ NMR $\left(250.12 \mathrm{MHz}, \mathrm{CD}_{3} \mathrm{CN}\right): \delta 1.20(\mathrm{t}, 3 \mathrm{H}, J 7.1 \mathrm{~Hz}$, $\left.\mathrm{CH}_{3}\right), 1.28,1.33\left[2 \mathrm{~s}\right.$, each $\left.6 \mathrm{H}, 2 \times \mathrm{C}\left(\mathrm{CH}_{3}\right)_{3}\right], 1.37,1.42[2 \mathrm{~s}$, each $\left.3 \mathrm{H}, \mathrm{C}\left(\mathrm{CH}_{3}\right)_{3}\right], 2.44,2.55\left[2 \mathrm{~m}\right.$, each $\left.2 \mathrm{H},\left(\mathrm{CH}_{2}\right)_{2}\right], 3.43$, $3.47\left(2 \mathrm{~s}\right.$, each $\left.3 \mathrm{H}, 2 \times \mathrm{OCH}_{3}\right), 3.09\left(\mathrm{ddd}, 1 \mathrm{H}, J_{5^{\prime}, 6^{\prime} \mathrm{a}} 8.1 \mathrm{~Hz}\right.$, $\left.J_{6^{\prime} \mathrm{a}, \mathrm{NH}} 5.5 \mathrm{~Hz}, J_{6^{\prime} \mathrm{a}, 6^{\prime} \mathrm{b}} 13.8 \mathrm{~Hz}, \mathrm{H}-6^{\prime} \mathrm{a}\right), 3.30-3.41\left(\mathrm{~m}, 2 \mathrm{H}, \mathrm{H}-2^{\prime}\right.$,
$\left.\mathrm{OH}-2^{\prime}\right), 3.52\left(\mathrm{q}, 2 \mathrm{H}, J \mathrm{~Hz}, \mathrm{CH}_{3} \mathrm{CH}_{2} \mathrm{~N}\right), 3.60-3.78(\mathrm{~m}$, $\left.4 \mathrm{H}, \mathrm{OCH}_{2} \mathrm{CH}_{2} \mathrm{~N}, \mathrm{H}-5^{\prime}, \mathrm{H}-6^{\prime} \mathrm{b}\right), 3.82$ (dd, $1 \mathrm{H}, J_{3,4} 1.2 \mathrm{~Hz}$, $\left.J_{4,5} 4.8 \mathrm{~Hz}, \mathrm{H}-4\right), 3.90-4.12$ (m, 5H, H-3', H-4', H-3, H-6a, $\mathrm{H}-6 \mathrm{~b}), 4.18$ (m, 1H, H-5), 4.25 (t, 2H, J 6.1 Hz, CH $\mathrm{CHCO}_{2}$, $4.30\left(\mathrm{~d}, 1 \mathrm{H}, J_{1^{\prime}, 2^{\prime}} 8.2 \mathrm{~Hz}, \mathrm{H}-1^{\prime}\right), 4.34\left(\mathrm{~d}, 1 \mathrm{H}, J_{1,2} 6.5 \mathrm{~Hz}, \mathrm{H}-1\right)$, 4.40 (dd, 1H, J2,3 $7.1 \mathrm{~Hz}, \mathrm{H}-2$ ), 6.67 (bt, 1H, NH), 7.73 (d, 1H, Ar-H-6), 6.86, 7.85 ( $\mathrm{AA}^{\prime} \mathrm{XX}^{\prime}$ system, 4H, Ar-H-8, Ar-H-9, Ar-H-11, Ar-H-12), 8.15 (dd, 1H, J5,6 9.0 Hz, Ar-H-5), 8.37 (d, $\left.1 \mathrm{H}, J_{3,5} 2.4 \mathrm{~Hz}, \mathrm{Ar}-\mathrm{H}-3\right) ;{ }^{13} \mathrm{C} \mathrm{NMR}\left(62.9 \mathrm{MHz}, \mathrm{CD}_{3} \mathrm{CN}\right): \delta$ $12.4\left(\mathrm{CH}_{3}\right), 25.1,26.5,26.6,26.9,27.4,28.4\left[3 \times \mathrm{C}\left(\mathrm{CH}_{3}\right)_{3}\right]$, 29.1, $30.9\left[\left(\mathrm{CH}_{2}\right)_{2}\right], 40.9\left(\mathrm{C}-6^{\prime}\right), 46.4\left(\mathrm{CH}_{3} \mathrm{CH}_{2} \mathrm{~N}\right), 49.5$ $\left(\mathrm{OCH}_{2} \mathrm{CH}_{2} \mathrm{~N}\right), 56.0,58.0\left(2 \times \mathrm{OCH}_{3}\right), 66.1(\mathrm{C}-6), 62.3$ $\left(\mathrm{CH}_{2} \mathrm{OCO}\right), 74.6\left(\mathrm{C}-2^{\prime}\right), 72.7\left(\mathrm{C}-5^{\prime}\right), 75.0\left(\mathrm{C}-4^{\prime}\right), 77.4(\mathrm{C}-2)$, 77.6 (C-5), 77.8 (C-4), 78.4 (C-3), $80.1\left(\mathrm{C}-3^{\prime}\right), 104.0\left(\mathrm{C}-1^{\prime}\right)$, 108.2 (C-1), 109.4, 110.6, $110.7\left[3 \times \mathrm{C}\left(\mathrm{CH}_{3}\right)_{3}\right], 112.7,112.7,118.8$, 123.9, 126.8, 127.6, 127.7 (7 × Ar-CH), 134.1 (Ar-C-2), 144.8 (Ar-C-7), 148.2 (Ar-C-4), 153.3, 153.8 (Ar-C-1, Ar-C-10), 172.3, $173.7(2 \times \mathrm{C}=\mathrm{O})$. Anal. Calcd for $\mathrm{C}_{43} \mathrm{H}_{60} \mathrm{ClN}_{5} \mathrm{O}_{16}$ (938.41): C, 55.04; H, 6.44; N, 7.46. Found: C, 55.02; H, 6.42; N, 7.43.

2.5. Preparation of Protected GAD 13. A solution of 6 (1.0 g, $2.78 \mathrm{mmol}$ ), azo dye 8 (950 mg, $3.52 \mathrm{mmol}, 1.25 \mathrm{eq}), N, N^{\dagger}$ dicyclohexylcarbodiimide (DDC) $(688 \mathrm{mg}, 3.34 \mathrm{mmol})$, and 4-dimethylaminopyridine (DMAP) $(102 \mathrm{mg}, 0.83 \mathrm{mmol})$ in dry THF $(30 \mathrm{~mL})$ was allowed to react in the reaction conditions described for the preparation of 11. The flash chromatography over silica gel (1:1 hexane-EtOAc) afforded pure $13\left(1.47 \mathrm{~g}, 86 \%\right.$ yield) as a yellow foam; $R_{f} 0.31$ (4:6 hexaneEtOAc); $\lambda_{\max } 407.00 \mathrm{~nm}(\mathrm{MeOH}) ;{ }^{1} \mathrm{H}$ NMR $(200.13 \mathrm{MHz}$, $\left.\mathrm{CDCl}_{3}\right): \delta 1.22\left(\mathrm{t}, 3 \mathrm{H}, J 7.0 \mathrm{~Hz}, \mathrm{CH}_{3}\right), 1.30,1.33,1.44,1.48[4 \mathrm{~s}$, each $\left.3 \mathrm{H}, 2 \times \mathrm{C}\left(\mathrm{CH}_{3}\right)_{3}\right], 2.47,2.66\left[2 \mathrm{~m}\right.$, each $\left.2 \mathrm{H},\left(\mathrm{CH}_{2}\right)_{2}\right]$, 3.20 (ddd, $1 \mathrm{H}, J_{6 \mathrm{a}, 6 \mathrm{~b}} 14.0 \mathrm{~Hz}, J_{5,6 \mathrm{a}} 9.0 \mathrm{~Hz}, J_{6 \mathrm{a}, \mathrm{NH}} 3.8 \mathrm{~Hz}, \mathrm{H}-$ 6a), 3.49 (q, $2 \mathrm{H}, J 7.0 \mathrm{~Hz}, \mathrm{CH}_{3} \mathrm{CH}_{2} \mathrm{~N}$ ), $3.64(\mathrm{t}, 2 \mathrm{H}, J 6.2 \mathrm{~Hz}$, $\mathrm{OCH}_{2} \mathrm{CH}_{2} \mathrm{~N}$ ), 3.71 (ddd, $1 \mathrm{H}, J_{5,6 \mathrm{~b}} 3.5 \mathrm{~Hz}, J_{6 \mathrm{~b}, \mathrm{NH}} 7.7 \mathrm{~Hz}, \mathrm{H}-6 \mathrm{~b}$ ), 3.87 (ddd, $1 \mathrm{H}, \mathrm{H}-5$ ), 4.18 (dd, $1 \mathrm{H}, J_{3,4} 7.9 \mathrm{~Hz}, J_{4,5} 1.8 \mathrm{~Hz}, \mathrm{H}-4$ ), $4.30\left(\mathrm{dd}, 1 \mathrm{H}, J_{1,2} 5.0 \mathrm{~Hz}, J_{2,3} 2.4 \mathrm{~Hz}, \mathrm{H}-2\right), 4.28(\mathrm{t}, 2 \mathrm{H}, J 6.2 \mathrm{~Hz}$, $\mathrm{CH}_{2} \mathrm{OCO}$ ), 4.57 (dd, $1 \mathrm{H}, \mathrm{H}-3$ ), 5.50 (d, 1H, H-1), 6.00 (dd, $1 \mathrm{H}$, $\mathrm{NH}), 6.78$ (m, 2H, Ar-H-9, Ar-H-11), 7.37-7.51 (m, 3H, Ar-H3, Ar-H-4, Ar-H-5), 7.81-7.90 (m, 4H, Ar-H-2, Ar-H-6, Ar-H8, Ar-H-12); ${ }^{13} \mathrm{CNMR}\left(50.3 \mathrm{MHz}, \mathrm{CDCl}_{3}\right): \delta 12.2\left(\mathrm{CH}_{3}\right), 24.3$, 24.9, 25.8, $25.9\left[2 \times \mathrm{C}\left(\mathrm{CH}_{3}\right)_{3}\right], 29.3,30.7\left[\left(\mathrm{CH}_{2}\right)_{2}\right], 40.0(\mathrm{C}-$ 6), $45.5\left(\mathrm{CH}_{3} \mathrm{CH}_{2} \mathrm{~N}\right), 48.7\left(\mathrm{OCH}_{2} \mathrm{CH}_{2} \mathrm{~N}\right), 61.5\left(\mathrm{CH}_{2} \mathrm{OCO}\right)$, 66.3 (C-5), 70.5 (C-2), 70.7 (C-3), 71.6 (C-4), 96.2 (C-1), 108.7, $109.4\left[2 \times \mathrm{C}\left(\mathrm{CH}_{3}\right)_{3}\right], 111.3,122.1,125.2,126.4,128.9(\mathrm{Ar}-\mathrm{CH})$, 143.6 (Ar-C-7), 149.9, 153.0, (Ar-C-1, Ar-C-10), 171.4, 172.7 (2× $\mathrm{C}=\mathrm{O}$ ). Anal. Calcd for $\mathrm{C}_{32} \mathrm{H}_{42} \mathrm{~N}_{4} \mathrm{O}_{8}$ (610.70): C, 62.94; $\mathrm{H}$, 6.93; N, 9.17. Found: C, 62.91; H, 6.90; N, 9.15.

2.6. Preparation of Protected GAD 14. A solution of azo dye 10 (152 mg, $0.367 \mathrm{mmol}$ ) and amine 4 (114 mg, $0.441 \mathrm{mmol}$, $1.2 \mathrm{eq})$ in dry THF $(4 \mathrm{~mL})$ was stirred at room temperature (10 min). 4-(4,6-Dimethoxy-1,3,5-triazin-2-yl)-4methylmorpholinium chloride (DMTMM) [13] (122 mg, $0.441 \mathrm{mmol}, 1.2 \mathrm{eq}$ ) was added and the mixture was stirred at room temperature until azo dye $\mathbf{1 0}$ was completely disappeared $(20 \mathrm{~h}, \mathrm{TLC}, \mathrm{EtOAc})$. The reaction mixture was concentrated under diminished pressure and the crude residue 
was partitioned between water $(10 \mathrm{~mL})$ and $\mathrm{Et}_{2} \mathrm{O}(10 \mathrm{~mL})$ and the aqueous phase extracted with $\mathrm{Et}_{2} \mathrm{O}(3 \times 10 \mathrm{~mL})$. The collected organic phases were dried $\left(\mathrm{Na}_{2} \mathrm{SO}_{4}\right)$, filtered, and concentrated under diminished pressure. Purification of the crude residue ( $3.10 \mathrm{~g}$ ) by flash chromatography over silica gel (1:4 hexane-EtOAc) gave pure 14 as a red foam solid (230 mg, 95\% yield calculated from 10); $R_{f} 0.45$ (EtOAc); ${ }^{1} \mathrm{H}$ NMR (250.12 MHz, $\left.\mathrm{CD}_{3} \mathrm{CN}\right): \delta 1.19\left(\mathrm{t}, 3 \mathrm{H}, J 7.0 \mathrm{~Hz}, \mathrm{CH}_{3}\right), 1.26,1.29$, $1.37,1.41\left[4 \mathrm{~s}\right.$, each $\left.3 \mathrm{H}, 2 \times \mathrm{C}\left(\mathrm{CH}_{3}\right)_{3}\right], 2.38,2.52[2 \mathrm{~m}$, each $2 \mathrm{H}$, $\left.\left(\mathrm{CH}_{2}\right)_{2}\right], 3.12$ (ddd, $1 \mathrm{H}, J_{6 \mathrm{a}, 6 \mathrm{~b}} 13.7 \mathrm{~Hz}, J_{5,6 \mathrm{a}} 8.1 \mathrm{~Hz}, J_{6 \mathrm{a}, \mathrm{NH}} 5.5 \mathrm{~Hz}$, H-6a), 3.36 (ddd, $1 \mathrm{H}, J_{5,6 \mathrm{~b}} 5.2 \mathrm{~Hz}, J_{6 \mathrm{~b}, \mathrm{NH}} 6.4 \mathrm{~Hz}, \mathrm{H}-6 \mathrm{~b}$ ), 3.54 (q, $\left.2 \mathrm{H}, J 7.0 \mathrm{~Hz}, \mathrm{CH}_{3} \mathrm{CH}_{2} \mathrm{~N}\right), 3.68\left(\mathrm{t}, 2 \mathrm{H}, J 5.9 \mathrm{~Hz}, \mathrm{CH}_{2} \mathrm{CH}_{2} \mathrm{~N}\right)$, 3.84 (ddd, 1H, H-5), 4.17 (dd, $1 \mathrm{H}, J_{3,4} 7.9 \mathrm{~Hz}, J_{4,5} 1.9 \mathrm{~Hz}, \mathrm{H}-$ 4), 4.29 (dd, $\left.1 \mathrm{H}, J_{1,2} 4.9 \mathrm{~Hz}, J_{2,3} 2.5 \mathrm{~Hz}, \mathrm{H}-2\right), 4.26$ (t, $2 \mathrm{H}, J$ $5.9 \mathrm{~Hz}, \mathrm{CH}_{2} \mathrm{OCO}$ ), 4.57 (dd, 1H, H-3), 5.41 (d, 1H, H-1), 6.52 (bt, $1 \mathrm{H}, \mathrm{NH}), 6.89,7.86$ ( $\mathrm{AA}^{\prime} \mathrm{XX}^{\prime}$ system, $4 \mathrm{H}, \mathrm{Ar}-\mathrm{H}-8, \mathrm{Ar}-\mathrm{H}-$ 9, Ar-H-11, Ar-H-12), 7.91, 8.31 ( $\mathrm{AA}^{\prime} \mathrm{XX}^{\prime}$ system, 4H, Ar-H2, Ar-H-3, Ar-H-5, Ar-H-6); ${ }^{13} \mathrm{C}$ NMR (62.9 MHz, CD $\left.3 \mathrm{CN}\right)$ : $\delta 12.4\left(\mathrm{CH}_{3}\right), 24.5,25.2,26.2,26.3\left[2 \times \mathrm{C}\left(\mathrm{CH}_{3}\right)_{3}\right], 30.0,30.9$ $\left[\left(\mathrm{CH}_{2}\right)_{2}\right], 40.6(\mathrm{C}-6), 46.3\left(\mathrm{CH}_{3} \mathrm{CH}_{2} \mathrm{~N}\right), 49.4\left(\mathrm{CH}_{2} \mathrm{CH}_{2} \mathrm{~N}\right)$, $62.3\left(\mathrm{CH}_{2} \mathrm{OCO}\right), 66.9$ (C-5), 71.4 (C-2), 71.5 (C-3), $72.2(\mathrm{C}-$ 4), $96.1(\mathrm{C}-1), 109.3,109.8\left[2 \times \mathrm{C}\left(\mathrm{CH}_{3}\right)_{3}\right], 112.6,123.3,125.7$, 126.9 (8 × Ar-CH), 144.3 (Ar-C-7), 148.4 (Ar-C-4), 152.7, 157.7 (Ar-C-1, Ar-C-10), 172.4, $173.6(2 \times \mathrm{C}=\mathrm{O})$. Anal. Calcd for $\mathrm{C}_{32} \mathrm{H}_{41} \mathrm{~N}_{5} \mathrm{O}_{10}$ (655.70): C, 58.62; H, 6.30; N, 10.68. Found: C, $58.60 ; \mathrm{H}, 6.28 ; \mathrm{N}, 10.65$.

2.7. Preparation of Protected GAD 15. The condensation of azo dye 10 (151 mg, $0.367 \mathrm{mmol})$ and amine 5 (224 mg, $0.441 \mathrm{mmol}, 1.2 \mathrm{eq})$ with 4-(4,6-dimethoxy-1,3,5-triazin2-yl)-4-methylmorpholinium chloride (DMTMM) [13] (122 mg, $0441 \mathrm{mmol}, 1.2 \mathrm{eq})$ in dry THF $(4 \mathrm{~mL})$ was performed according to the procedure described above for the preparation of $\mathbf{1 4}$. The flash chromatography over silica gel (1:9 hexane-EtOAc) of the crude reaction product led to pure 15 (252 mg, 76\% yield calculated from 10) as a red foam; $R_{f} 0.32$ (EtOAc); ${ }^{1} \mathrm{H}$ NMR $\left(250.12 \mathrm{MHz}, \mathrm{CD}_{3} \mathrm{CN}\right): \delta 1.18(\mathrm{t}$, $\left.3 \mathrm{H}, J 7.0 \mathrm{~Hz}, \mathrm{CH}_{3}\right), 1.27,1.33$ [2s, each $6 \mathrm{H}, 2 \times \mathrm{C}\left(\mathrm{CH}_{3}\right)_{3}$ ], 1.37, $1.42\left[2 \mathrm{~s}\right.$, each $\left.3 \mathrm{H}, \mathrm{C}\left(\mathrm{CH}_{3}\right)_{3}\right], 2.44,2.55[2 \mathrm{~m}$, each $2 \mathrm{H}$, $\left.\left(\mathrm{CH}_{2}\right)_{2}\right], 3.43,3.46\left(2 \mathrm{~s}\right.$, each $\left.3 \mathrm{H}, 2 \times \mathrm{OCH}_{3}\right), 3.11(\mathrm{ddd}, 1 \mathrm{H}$, $\left.J_{5^{\prime}, 6^{\prime} \mathrm{a}} 8.8 \mathrm{~Hz}, J_{6^{\prime} \mathrm{a}, \mathrm{NH}} 5.2 \mathrm{~Hz}, J_{6^{\prime} \mathrm{a}, 6^{\prime} \mathrm{b}} 13.4 \mathrm{~Hz}, \mathrm{H}-6^{\prime} \mathrm{a}\right), 3.32$ (dd, $\left.1 \mathrm{H}, J_{1^{\prime}, 2^{\prime}} 8.0 \mathrm{~Hz}, J_{2^{\prime}, 3^{\prime}} 7.3 \mathrm{~Hz}, \mathrm{H}-2^{\prime}\right), 3.51(\mathrm{q}, 2 \mathrm{H}, J 7.0 \mathrm{~Hz}$, $\left.\mathrm{CH}_{3} \mathrm{CH}_{2} \mathrm{~N}\right), 3.67\left(\mathrm{~m}, 2 \mathrm{H}, J 6.0 \mathrm{~Hz}, \mathrm{CH}_{2} \mathrm{CH}_{2} \mathrm{~N}\right), 3.70-3.75$ (m, 3H, H-5', H-6'b, OH-2'), $3.82\left(\mathrm{dd}, 1 \mathrm{H}, J_{4,5} 5.2 \mathrm{~Hz}, J_{3,4}\right.$ $1.3 \mathrm{~Hz}, \mathrm{H}-4), 3.94$ (dd, $1 \mathrm{H}, J_{3^{\prime}, 4^{\prime}} 3.4 \mathrm{~Hz}, \mathrm{H}-3^{\prime}$ ), 4.00 (dd, $1 \mathrm{H}$, $J_{6 \mathrm{a}, 6 \mathrm{~b}} 8.1 \mathrm{~Hz}, J_{5,6 \mathrm{a}} 6.3 \mathrm{~Hz}, \mathrm{H}-6 \mathrm{a}$ ), 4.03 (dd, $1 \mathrm{H}, J_{2,3} 7.2 \mathrm{~Hz}, \mathrm{H}-3$ ), 4.04-4.10 (m, 2H, H-4', H-6b), $4.22\left(\mathrm{dt}, 1 \mathrm{H}, J_{5,6 \mathrm{a}}=J_{5,6 \mathrm{~b}}\right.$ $6.3 \mathrm{~Hz}, \mathrm{H}-5), 4.27$ (t, 2H, J 6.0 Hz, $\left.\mathrm{CH}_{2} \mathrm{OCO}\right), 4.31$ (d, $1 \mathrm{H}, \mathrm{H}-$ $\left.1^{\prime}\right), 4.35$ (d, $\left.1 \mathrm{H}, J_{1,2} 6.4 \mathrm{~Hz}, \mathrm{H}-1\right), 4.41$ (dd, $\left.1 \mathrm{H}, \mathrm{H}-2\right), 6.68$ (bt, $1 \mathrm{H}, \mathrm{NH}), 6.85,7.68\left(\mathrm{AA}^{\prime} \mathrm{XX}^{\prime}\right.$ system, 4H, Ar-H-8, Ar-H-9, Ar-H-11, Ar-H-12), 7.88, 8.29 ( $\mathrm{AA}^{\prime} \mathrm{XX}^{\prime}$ system, 4H, Ar-H-2, Ar-H-3, Ar-H-5, Ar-H-6); ${ }^{13} \mathrm{C}$ NMR (62.9 MHz, CD 3 CN): $\delta 12.4\left(\mathrm{CH}_{3}\right), 25.1,26.4,26.5,26.9,27.4,28.4\left[3 \times \mathrm{C}\left(\mathrm{CH}_{3}\right)_{3}\right]$, 30.9, $29.9\left[\left(\mathrm{CH}_{2}\right)_{2}\right], 40.9\left(\mathrm{C}-6^{\prime}\right), 46.2\left(\mathrm{CH}_{3} \mathrm{CH}_{2} \mathrm{~N}\right), 49.5$ $\left(\mathrm{OCH}_{2} \mathrm{CH}_{2} \mathrm{~N}\right), 56.0,58.0\left(2 \times \mathrm{OCH}_{3}\right), 62.3\left(\mathrm{CH}_{2} \mathrm{OCO}\right), 66.0$ (C-6), $74.6\left(\mathrm{C}-2^{\prime}\right), 72.6\left(\mathrm{C}-5^{\prime}\right), 75.0\left(\mathrm{C}-4^{\prime}\right), 77.4(\mathrm{C}-2), 77.6$

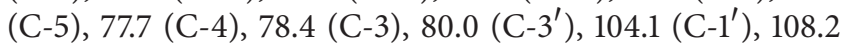

(C-1), 109.2, 110.4, $110.6\left[3 \times \mathrm{C}\left(\mathrm{CH}_{3}\right)_{3}\right], 112.6,123.3,125.7$, 126.9 (8 × Ar-CH), 144.2 (Ar-C-7), 148.3 (Ar-C-4), 152.7, 157.6 (Ar-C-1, Ar-C-10), 172.3, $173.7(2 \times \mathrm{C}=\mathrm{O})$. Anal. Calcd for $\mathrm{C}_{43} \mathrm{H}_{61} \mathrm{~N}_{5} \mathrm{O}_{16}$ (903.37): C, 57.13; $\mathrm{H}, 6.80$; N, 7.75. Found: C, 57.11; H, 6.77; N, 7.72.

2.8. Preparation of Deprotected GAD 16. A solution of $\mathbf{1 1}$ $(1.07 \mathrm{~g}, 1.55 \mathrm{mmol})$ in $90 \%$ aqueous $\mathrm{CF}_{3} \mathrm{COOH}(70 \mathrm{~mL})$ was stirred at room temperature until the starting material was completely disappeared ( $3 \mathrm{~h}, \mathrm{TLC}$, EtOAc). The red-violet solution was concentrated under diminished pressure and repeatedly coevaporated with toluene $(5 \times 30 \mathrm{~mL})$. The crude residue was dissolved in EtOAc $(100 \mathrm{~mL})$ and neutralized with saturated aqueous $\mathrm{NaHCO}_{3}(20 \mathrm{~mL})$. The aqueous phase was extracted with EtOAc $(3 \times 50 \mathrm{~mL})$ and the collected layers were dried, filtered, and concentrated under diminished pressure. The residue was triturated with $\mathrm{Et}_{2} \mathrm{O}$ and the red residue was constituted (NMR) only by the azo dye $\mathbf{1 6}$ (640 mg, 98\% yield) as a mixture of $\alpha$ - and $\beta$-pyranose anomers (NMR) in a ratio of $3: 2$, calculated on the basis of the relative C-1 signal intensities. Compound $\mathbf{1 6}$ was a red solid foam, $R_{f} 0.21$ (9:1 EtOAc-MeOH); Selected ${ }^{1} \mathrm{H}$ NMR $\left(200.13 \mathrm{MHz}, \mathrm{CD}_{3} \mathrm{OD}\right)$ signals for both anomers: $\delta 1.06(\mathrm{t}$, $\left.3 \mathrm{H}, J 6.7 \mathrm{~Hz}, \mathrm{CH}_{3}\right), 2.48-2.30\left[\mathrm{~m}, 4 \mathrm{H},\left(\mathrm{CH}_{2}\right)_{2}\right], 6.73,7.64$, ( $\mathrm{AA}^{\prime} \mathrm{XX}^{\prime}$ system, 4H, Ar-H-8, Ar-H-9, Ar-H-11, Ar-H-12), 7.54 (bd, $1 \mathrm{H}, \mathrm{Ar}-\mathrm{H}-6), 8.00$ (dd, $\left.1 \mathrm{H}, J_{5,6} 9.0 \mathrm{~Hz}, \mathrm{Ar}-\mathrm{H}-5\right), 8.40$ (d, $\left.1 \mathrm{H}, J_{3,5} 2.2 \mathrm{~Hz}, \mathrm{Ar}-\mathrm{H}-3\right) ;{ }^{13} \mathrm{C} \mathrm{NMR}\left(50.3 \mathrm{MHz}, \mathrm{CD}_{3} \mathrm{OD}\right): \delta$ $\alpha$-pyranose 40.8 (C-6), 68.8 (C-5), 69.2 (C-4), 69.7 (C-2, C-3), 93.8 (C-1), $\beta$-pyranose 40.7 (C-6), 69.1 (C-4), 72.5 (C-3), 73.0, 73.7 (C-2, C-5), 97.8 (C-1), cluster of signals for both anomers: $\delta 12.5\left(\mathrm{CH}_{3}\right), 29.4-30.9\left[\left(\mathrm{CH}_{2}\right)_{2}\right], 45.9\left(\mathrm{CH}_{3} \mathrm{CH}_{2} \mathrm{~N}\right), 48.9$ $\left(\mathrm{OCH}_{2} \mathrm{CH}_{2} \mathrm{~N}\right), 62.0\left(\mathrm{CH}_{2} \mathrm{OCO}\right), 112.2-127.2(\mathrm{Ar}-\mathrm{CH}), 133.0$ (Ar-C-2), 143.9 (Ar-C-7), 147.0 (Ar-C-4), 152.6, 152.8 (Ar-C-1, Ar-C-10), 172.4, $173.2(2 \times \mathrm{C}=\mathrm{O})$. Calcd for $\mathrm{C}_{26} \mathrm{H}_{32} \mathrm{ClN}_{5} \mathrm{O}_{10}$ (610.01): C, 51.19; H, 5.29; N, 11.48. Found: C, 51.17; H, 5.28; N, 11.45 .

2.9. Preparation of Deprotected GAD 17. Hydrolysis of $\mathbf{1 2}$ (640 mg, $0.682 \mathrm{mmol}$ ) with $90 \%$ aqueous $\mathrm{CF}_{3} \mathrm{COOH}(8 \mathrm{~mL})$ was performed as described above for the preparation of $\mathbf{1 6}$. After $2 \mathrm{~h}$ of stirring, the red-violet solution was concentrated under diminished pressure, neutralized, and extracted as described above to give a red foam constituted (NMR) only by the azo dye 17 ( $485 \mathrm{mg}, 92 \%$ yield) as a mixture of $\alpha$ - and $\beta$-pyranose anomers (NMR) in the ratio of $3: 2$, calculated on the basis of the relative C-1 signal intensities. Compound $\mathbf{1 7}$ was a red solid foam, $R_{f} 0.18$ (8:2 EtOAc-MeOH); $\lambda_{\max }$ $493.25 \mathrm{~nm}$ (MeOH); Selected ${ }^{1} \mathrm{H}$ NMR $(200.13 \mathrm{MHz}$, $\left.\mathrm{CD}_{3} \mathrm{OD}\right): \delta 1.20\left(\mathrm{t}, 3 \mathrm{H}, J 7.1 \mathrm{~Hz}, \mathrm{CH}_{3}, \alpha\right.$ - and $\beta$-pyranose), 2.48, 2.57 [ $2 \mathrm{~m}$, each $2 \mathrm{H},\left(\mathrm{CH}_{2}\right)_{2}, \alpha$ - and $\beta$-pyranose], 3.18 (dd, $1 \mathrm{H}, J_{1,2} 7.8 \mathrm{~Hz}, J_{2,3} 9.0 \mathrm{~Hz}, \mathrm{H}$-2, $\beta$-pyranose), 4.18 (d, $1 \mathrm{H}, J_{1^{\prime}, 2^{\prime}} 8.0 \mathrm{~Hz}, \mathrm{H}-1^{\prime}, \alpha$-pyranose), $4.20\left(\mathrm{~d}, 1 \mathrm{H}, J_{1^{\prime}, 2^{\prime}} 7.8 \mathrm{~Hz}\right.$, $\mathrm{H}-1^{\prime}, \beta$-pyranose), 4.25 (bt, $2 \mathrm{H}, J 6.4 \mathrm{~Hz}, \mathrm{CH}_{2} \mathrm{OCO}, \alpha-$ and $\beta$-pyranose), 4.31 (bt, $2 \mathrm{H}, J 6.6 \mathrm{~Hz}, \mathrm{CH}_{2} \mathrm{OCO}, \alpha$ - and $\beta$-pyranose), 4.48 (d, $1 \mathrm{H}, \mathrm{H}-1, \beta$-pyranose), 4.49-4.61 (m, $\mathrm{H}-2^{\prime}, \alpha$ - and $\beta$-pyranose), 5.06 (d, $1 \mathrm{H}, J_{1,2} 3.7 \mathrm{~Hz}, \mathrm{H}-1, \alpha-$ pyranose), 6.85, 7.83 ( $\mathrm{AA}^{\prime} \mathrm{XX}^{\prime}$ system, $4 \mathrm{H}, \mathrm{Ar}-\mathrm{H}-8, \mathrm{Ar}-\mathrm{H}-9$, 


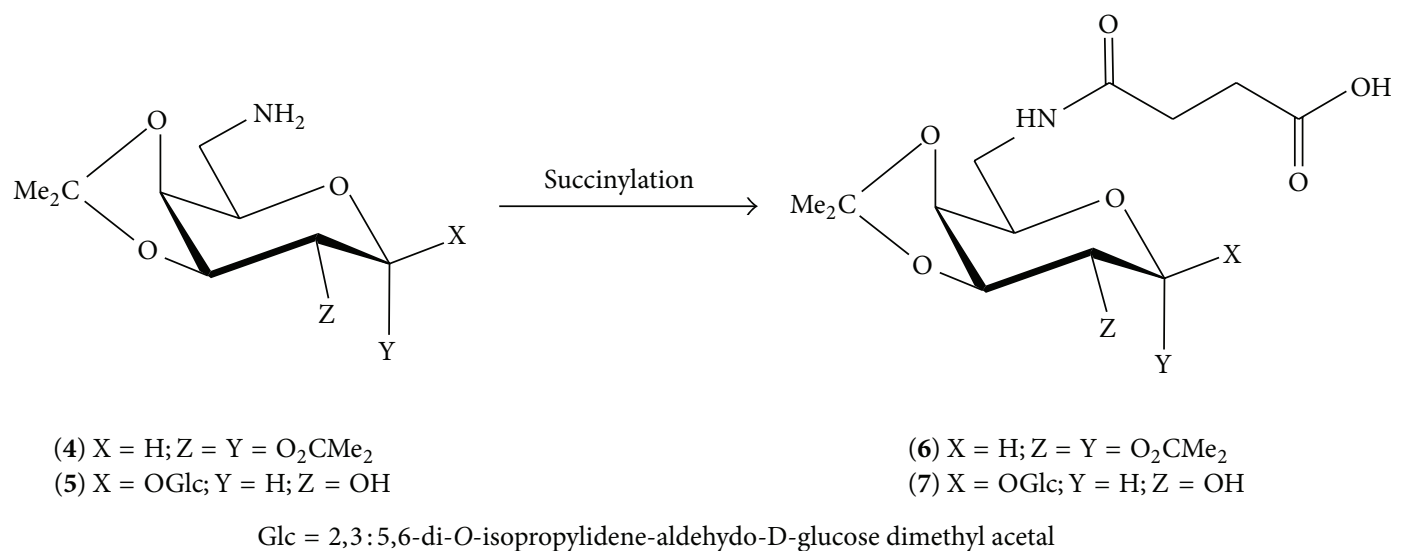

Scheme 1: Protected aminosugars (4 and 5) and their monosuccinylamides (6 and 7).

Ar-H-11, Ar-H-12, $\alpha$ - and $\beta$-pyranose), 7.72 (bd, 1H, Ar-H-6, $\alpha$ - and $\beta$-pyranose), 8.13 (bd, $1 \mathrm{H}, J 9.1 \mathrm{~Hz}, \mathrm{Ar}-\mathrm{H}-5, \alpha$ - and $\beta$-pyranose), 8.32 (bd, $1 \mathrm{H}, \mathrm{Ar}-\mathrm{H}$-3, $\alpha$ - and $\beta$-pyranose); ${ }^{13} \mathrm{C}$ NMR $\left(62.9 \mathrm{MHz}, \mathrm{CD}_{3} \mathrm{OD}\right): \delta \alpha$-pyranose $41.3\left(\mathrm{C}-6^{\prime}\right), 62.0$ (C-6), $70.5\left(\mathrm{C}-4^{\prime}\right), 71.4(\mathrm{C}-5), 72.1\left(\mathrm{C}-2^{\prime}\right), 72.4(\mathrm{C}-2), 73.0$ (C-3), $73.4\left(\mathrm{C}-3^{\prime}\right), 76.0\left(\mathrm{C}-5^{\prime}\right), 81.1(\mathrm{C}-4), 93.7\left(\mathrm{C}-1^{\prime}\right), 105.0$ (C-1), $\beta$-pyranose $41.2\left(\mathrm{C}-6^{\prime}\right), 62.8$ (C-6), $70.4\left(\mathrm{C}-4^{\prime}\right), 72.3$ $\left(\mathrm{C}-2^{\prime}\right), 73.1\left(\mathrm{C}-3^{\prime}\right), 74.5,74.6$ (C-3, C-2), 74.7 (C-5), 76.2 $\left(\mathrm{C}-5^{\prime}\right), 80.5(\mathrm{C}-4), 98.1\left(\mathrm{C}-1^{\prime}\right), 105.3(\mathrm{C}-1)$, cluster of signals for both anomers: $\delta 12.5\left(\mathrm{CH}_{3}\right), 30.3,31.3\left[\left(\mathrm{CH}_{2}\right)_{2}\right], 46.7$ $\left(\mathrm{CH}_{3} \mathrm{CH}_{2} \mathrm{~N}\right), 50.0\left(\mathrm{OCH}_{2} \mathrm{CH}_{2} \mathrm{~N}\right), 62.9\left(\mathrm{CH}_{2} \mathrm{OCO}\right), 112.9-$ $129.9(7 \times \mathrm{Ar}-\mathrm{CH}), 134.7$ (Ar-C-2), 145.6 (Ar-C-7), 148.6 (Ar-C-4), 153.6, 154.3 (Ar-C-1, Ar-C-10), 174.4, $174.9(2 \times$ $\mathrm{C}=\mathrm{O}$ ). Anal. Calcd for $\mathrm{C}_{32} \mathrm{H}_{42} \mathrm{ClN}_{5} \mathrm{O}_{15}$ (772.15): C, 49.78; $\mathrm{H}$, 5.48; N, 9.07. Found: C, 49.75; H, 5.46; N, 9.05.

2.10. Preparation of Deprotected GAD 18. The hydrolysis of $\mathbf{1 3}$ $(193 \mathrm{mg}, 0.316 \mathrm{mmol})$ with $90 \%$ aqueous $\mathrm{CF}_{3} \mathrm{COOH}(3 \mathrm{~mL})$ was performed as described above for the preparation of $\mathbf{1 6}$. After $2 \mathrm{~h}$ of stirring, the red-violet solution was concentrated under diminished pressure, neutralized (saturated aqueous $\mathrm{NaHCO}_{3}$ ) until the red-violet colour turned to yelloworange, and extracted as described above to give a yellow foam constituted (NMR) only by the azo dye 18 (160 mg, 95\% yield). Compound $\mathbf{1 8}$ was a mixture of $\alpha$ - and $\beta$-pyranose anomers (NMR) in the ratio of $1: 1$, calculated on the basis of the relative C-1 signal intensities; $R_{f} 0.19$ (9:1 EtOAc-MeOH); $\lambda_{\max } 411.25 \mathrm{~nm}(\mathrm{MeOH})$; Selected ${ }^{1} \mathrm{H}$ NMR $(200.13 \mathrm{MHz}$, $\left.\mathrm{CD}_{3} \mathrm{OD}\right)$ signals for both anomers: $\delta 1.21(\mathrm{t}, 3 \mathrm{H}, J 6.1 \mathrm{~Hz}$, $\left.\mathrm{CH}_{3}\right), 2.58\left[\mathrm{~m}, 4 \mathrm{H},\left(\mathrm{CH}_{2}\right)_{2}\right], 6.83$ (m, 2H, Ar-H-9, Ar-H-11), 7.35-7.50 (m, 3H, Ar-H-3, Ar-H-4, Ar-H-5), 7.75-7.83 (m, 4H, Ar-H-2, Ar-H-6, Ar-H-8, Ar-H-12); ${ }^{13} \mathrm{C}$ NMR $(62.9 \mathrm{MHz}$, $\mathrm{CD}_{3} \mathrm{OD}$ ): $\delta \alpha$-pyranose $40.1(\mathrm{C}-6), 67.5(\mathrm{C}-5), 69.4,69.5$, 69.7 (C-2, C-3, C-4), 93.6 (C-1), $\beta$-pyranose 40.5 (C-6), 70.3 (C-4), 72.1 (C-3), 73.1, 74.1 (C-2, C-5), 98.2 (C-1), cluster of signals for both anomers: $\delta 11.9\left(\mathrm{CH}_{3}\right), 28.5,28.6\left[\left(\mathrm{CH}_{2}\right)_{2}\right]$, $46.2\left(\mathrm{CH}_{3} \mathrm{CH}_{2} \mathrm{~N}\right), 52.9\left(\mathrm{OCH}_{2} \mathrm{CH}_{2} \mathrm{~N}\right), 59.8\left(\mathrm{CH}_{2} \mathrm{OCO}\right), 111.1$, 122.5, 125.7, 129.5, 129.9 (Ar-CH), 143.2 (Ar-C-7), 150.1, 153.0 (Ar-C-1, Ar-C-10), 174.0, $174.2(2 \times \mathrm{C}=\mathrm{O})$. Anal. Calcd for
$\mathrm{C}_{26} \mathrm{H}_{34} \mathrm{~N}_{4} \mathrm{O}_{8}$ (530.57): C, 58.86; H, 6.46; N, 10.56. Found: C, 58.83; H, 6.42; N, 10.53 .

\section{Results and Discussion}

In the search of new derivatives to be tested as new GADs, we followed the same strategy used for the synthesis of the di-estereal [4, 5] (1) and diethereal [6] (2) compounds. We were interested in preparing structures with different functional groups at the attachment point with the linker in order to evaluate the synthetic accessibility and, at a later stage, the effect of these changes both on the stability during the dyeing process and on the tinctorial properties. To construct the amido-estereal mixed GADs, we used appropriately protected 6-amino-galactose derivatives in either the condensation reaction with monosuccinyl-azo dye or after their conversion into monosuccinyl derivatives. This would allow us to study the two complementary strategies and to identify the best pathway.

Treatment of 4 [10] and 5 [8] with succinic anhydride in methanol (Scheme 1) afforded, in good yields, the corresponding monosuccinyl derivatives 6 (76\% yield) and 7 ( $98 \%$ yield) that were utilized in the GAD synthesis via route A (Scheme 2). Azo dyes 8-10 (Figure 2) were employed as counterpart in the condensation reaction.

In particular, the known yellow azo dye (8) [12] and the commercial Disperse Red 13 (9) were used in the preparation of GADs following route A (Scheme 2) while derivative 10, prepared by succinylation of commercial alcoholic azo dye Disperse Red 1, [4] was employed in the condensation reaction with the aminosugar derivatives 4 [10] and 5 [8] (route B).

The coupling reactions were performed in THF with $N, N^{\prime}$-dicyclohexylcarbodiimide (DCC, method a) or 4-(4,6dimethoxy-1,3,5-triazin-2-yl)-4-methylmorpholinium chloride (DMTMM, method b), prepared according to Kunishima et al. [13] from 2-chloro-4,6-dimethoxy-1,3,5-triazine (CDMT) and $N$-methylmorpholine (NMM) at room temperature. The results obtained in the preparation of protected GADs 11-15 are summarised in Table 1. The two possible 
Route A

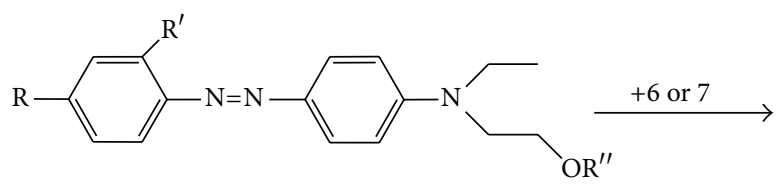

(8) $\mathrm{R}=\mathrm{R}^{\prime}=\mathrm{R}^{\prime \prime}=\mathrm{H}$

(9) $\mathrm{R}=\mathrm{NO}_{2} ; \mathrm{R}^{\prime}=\mathrm{Cl} ; \mathrm{R}^{\prime \prime}=\mathrm{H}$

Route B

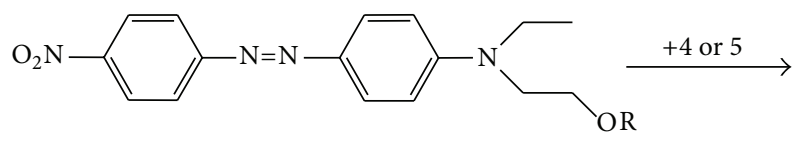

(10) $\mathrm{R}=\mathrm{CO}\left(\mathrm{CH}_{2}\right)_{2} \mathrm{COOH}$
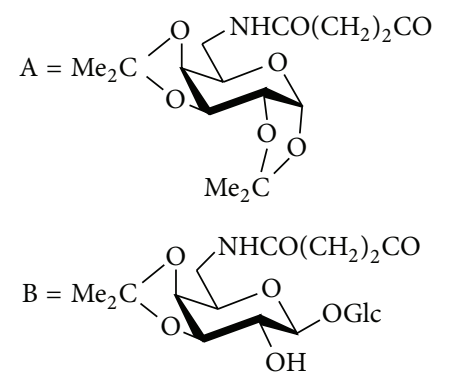

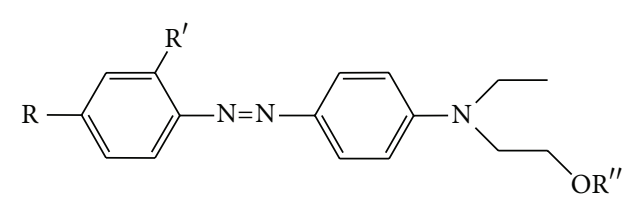

(11) $\mathrm{R}=\mathrm{NO}_{2} ; \mathrm{R}^{\prime}=\mathrm{Cl} ; \mathrm{R}^{\prime \prime}=\mathrm{A}$

(12) $\mathrm{R}=\mathrm{NO}_{2} ; \mathrm{R}^{\prime}=\mathrm{Cl} ; \mathrm{R}^{\prime \prime}=\mathrm{B}$

(13) $\mathrm{R}=\mathrm{R}^{\prime}=\mathrm{H} ; \mathrm{R}^{\prime \prime}=\mathrm{A}$

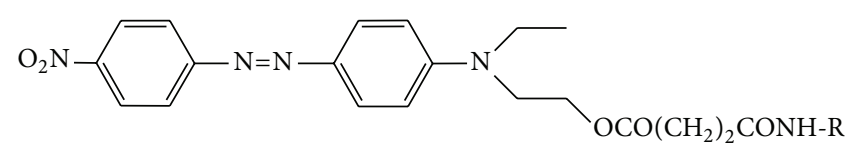

(14) $\mathrm{R}=\mathrm{C}$

(15) $\mathrm{R}=\mathrm{D}$

Scheme 2: Protected amido-ester GADs prepared through either method A or method B.

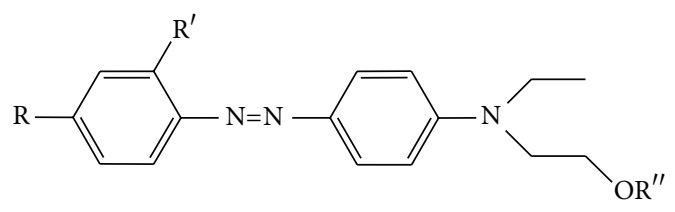

(8) $\mathrm{R}=\mathrm{R}^{\prime}=\mathrm{R}^{\prime \prime}=\mathrm{H}$

(9) $\mathrm{R}=\mathrm{NO}_{2} ; \mathrm{R}^{\prime}=\mathrm{Cl} ; \mathrm{R}^{\prime \prime}=\mathrm{H}$

(10) $\mathrm{R}=\mathrm{NO}_{2} ; \mathrm{R}^{\prime}=\mathrm{H} ; \mathrm{R}^{\prime \prime}=\mathrm{CO}\left(\mathrm{CH}_{2}\right)_{2} \mathrm{COOH}$

Figure 2: Monoazo dyes 8-9 and monosuccinyl-azo dye 10.

approaches gave comparable results and the desired products were isolated in good to excellent yields.

It is noteworthy that the succinyl derivatives $\mathbf{7}$ and $\mathbf{1 0}$ could be used as crudes without affecting the outcome of the condensation reaction. In the case of protected compounds 11-13 the final deprotected GADs 16-18 (Figure 3) were obtained by simply removing the acetal protecting groups by means of acid hydrolysis as we previously reported [4-6] for type 1 and type 2 derivatives (90\% aqueous $\mathrm{CF}_{3} \mathrm{COOH}$, room temperature).

Deprotected GADs were obtained in good yields (9298\%) as red (16-17) or yellow-orange (18) amorphous residues constituted by mixtures of anomers (NMR analysis).
Isomeric forms of the 16-18 were identified by comparison of their NMR data with those reported for analogous compounds (types 1-2) [4-6]. The UV-Vis absorption spectra for a deprotected red GAD (17) and for the deprotected yellow GAD (18) were recorded in $\mathrm{MeOH}$. As for the previous two generations of GADs, $[4,5]$ the glycoconjugation process did not affect the absorption properties of the dyes and $\mathbf{1 7}$ and 18 were characterised by almost the same $\lambda_{\max }$ values of the parent not conjugated dyes $\left(\lambda_{\max }\right.$ around $495 \mathrm{~nm}$ for the red compounds $9, \mathbf{1 2}$, and $\mathbf{1 7}$ and around $410 \mathrm{~nm}$ for the yelloworange compounds 8,13 , and 18 ).

\section{Conclusions}

In conclusions, we presented the access to the third generation of GADs exploring two complementary routes. The obtained compounds are characterized by different attachment points between the chromophore or the sugar and the linker. The presence of the amido bond could permit a novel synthetic pathway to GADs, for example, by using enzymes [14] for the condensation reaction. Detailed analysis of the stability and tinctorial properties of the third generation of GADs are currently under investigation, the results of which will be published in due course. 
TABLE 1: Preparation of amido-ester protected GADs.

\begin{tabular}{lcccc}
\hline Entry & \multicolumn{2}{c}{ Reactants } & Condensation system & Condensation product (yield\%) \\
\hline $\mathbf{1}$ & Sugar moiety & Dye moiety & Method A & $\mathbf{1 1}(74)$ \\
$\mathbf{2}$ & $\mathbf{6}$ & $\mathbf{9}$ & Method A & $\mathbf{1 2}(82)$ \\
$\mathbf{3}$ & $\mathbf{7}$ & $\mathbf{9}$ & Method A & $\mathbf{1 3}(86)$ \\
$\mathbf{4}$ & $\mathbf{6}$ & $\mathbf{8}$ & Method B & $\mathbf{1 4}(95)$ \\
$\mathbf{5}$ & $\mathbf{4}$ & $\mathbf{1 0}$ & Method B & $\mathbf{1 5}(76)$ \\
\hline
\end{tabular}

Method A: DCC, DMAP, and THF; method B: DMTMM and THF.

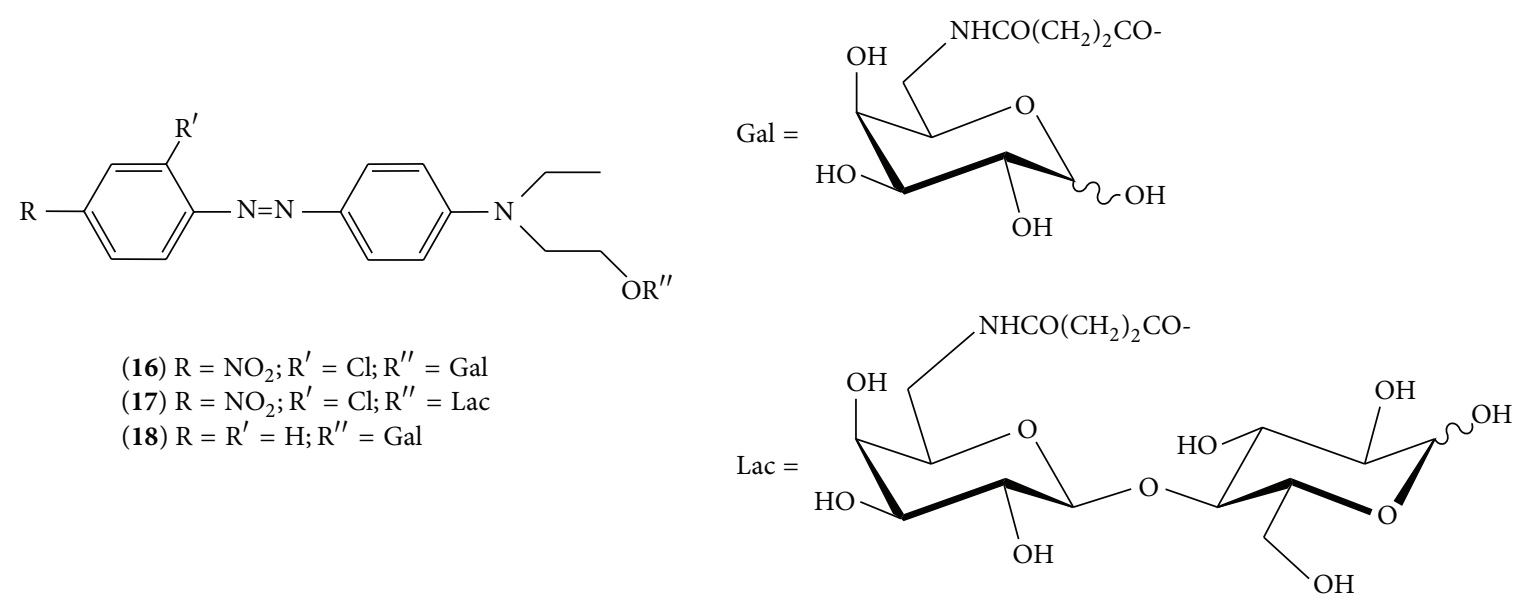

Figure 3: Conjugated deprotected GADs 16-18.

\section{Conflict of Interests}

The authors declare that there is no conflict of interests regarding the publication of this paper.

\section{References}

[1] H.-K. Rouette, Encyclopedia of Textile Finishing, Springer, Berlin, Germany, 2001.

[2] J. H. Hofenk de Graaf, The Colourful Past. Origins, Chemistry and Identification of Natural Dyestuffs, Abegg-Stitung, Riggsberg, Switzerland; Archetype Pubblications, London, UK, 2004.

[3] M. Neamtu, I. Siminiceanu, A. Yediler, and A. Kettrup, "Kinetics of decolorization and mineralization of reactive azo dyes in aqueous solution by the UV/ $\mathrm{H}_{2} \mathrm{O}_{2}$ oxidation," Dyes and Pigments, vol. 53, no. 2, pp. 93-99, 2002.

[4] G. Bartalucci, R. Bianchini, G. Catelani, F. D’Andrea, and L. Guazzelli, "Naturalised dyes: a simple straightforward synthetic route to a new class of dyes-glycoazodyes (GADs)," European Journal of Organic Chemistry, no. 4, pp. 588-595, 2007.

[5] R. Bianchini, G. Catelani, R. Cecconi et al., "Naturalization' of textile disperse dyes through glycoconjugation: the case of a bis(2-hydroxyethyl) group containing azo dye," Carbohydrate Research, vol. 343, no. 12, pp. 2067-2074, 2008.

[6] R. Bianchini, G. Catelani, R. Cecconi et al., "Ethereal glycoconjugated azodyes (GADs): a new group of water-soluble, naturalised dyes," European Journal of Organic Chemistry, no. 3, pp. 444-454, 2008.
[7] A. Porri, R. Baroncelli, L. Guglielminetti et al., "Fusarium oxysporum degradation and detoxification of a new textileglycoconjugate azo dye (GAD)," Fungal Biology, vol. 115, no. 1, pp. 30-37, 2011.

[8] J. Isaad, M. Rolla, and R. Bianchini, "Synthesis of water-soluble large naturalised dyes through double glycoconjugation," European Journal of Organic Chemistry, no. 17, pp. 2748-2764, 2009.

[9] R. Bianchini, M. Rolla, J. Isaad et al., "Efficient double glycoconjugation to naturalize high molecular weight disperse dyes," Carbohydrate Research, vol. 356, pp. 104-109, 2012.

[10] D. D. Perrin, W. L. F. D. Armarego, and R. Perrin, Purification of Laboratory Chemicals, Pergamon Press, Oxford, UK, 2nd edition, 1980.

[11] J. Yang, X. Fu, Q. Jia et al., "Studies on the substrate specificity of Escherichia coli galactokinase," Organic Letters, vol. 5, no. 13, pp. 2223-2226, 2003.

[12] H. Wahl, Y. Arnould, and M. Simon, "Dyes for cellulose acetate. II. Poly(hydroxyethyl)aniline dyes," Bulletin de la Societe Chimique de France, pp. 366-369, 1952.

[13] M. Kunishima, C. Kawachi, F. Iwasaki, K. Terao, and S. Tani, "Synthesis and characterization of 4-(4,6-dimethoxy1,3,5-triazin-2-yl)- 4-methylmorpholinium chloride," Tetrahedron Letters, vol. 40, no. 29, pp. 5327-5330, 1999.

[14] J. Sharma, D. Batovska, Y. Kuwamori, and Y. Asano, "Enzymatic chemoselective synthesis of secondary-amide surfactant from $\mathrm{N}$-methylethanol amine," Journal of Bioscience and Bioengineering, vol. 100, no. 6, pp. 662-666, 2005. 

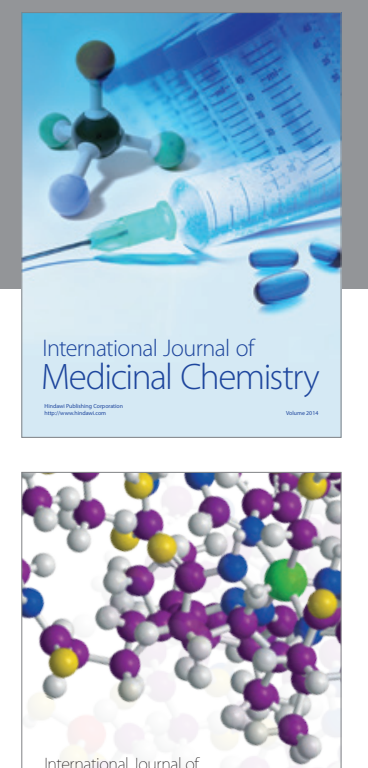

\section{Carbohydrate} Chemistry

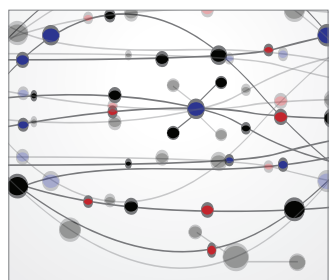

The Scientific World Journal
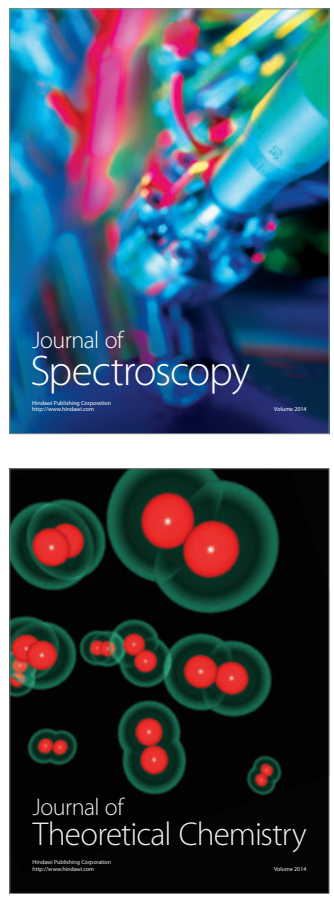
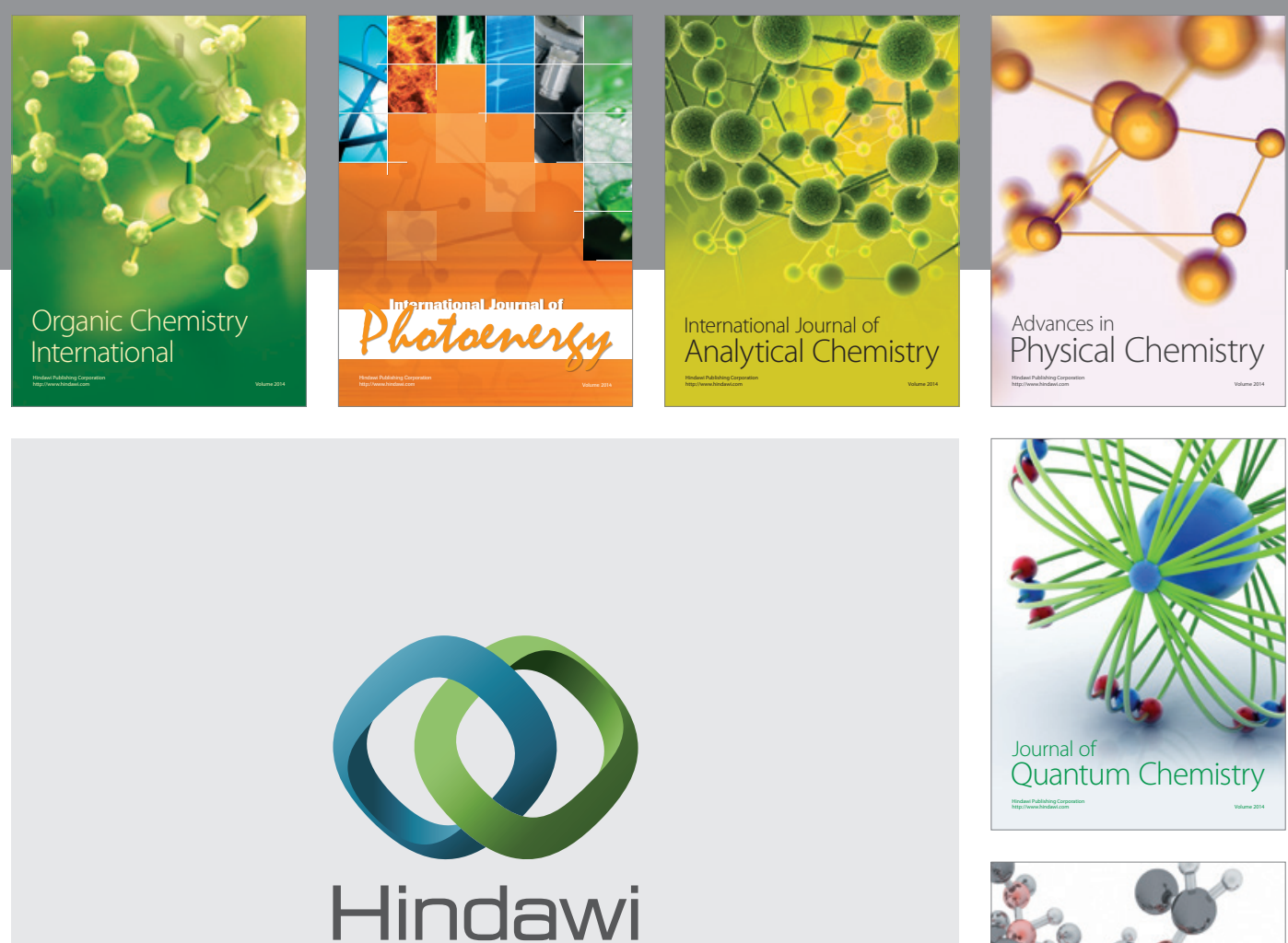

Submit your manuscripts at

http://www.hindawi.com

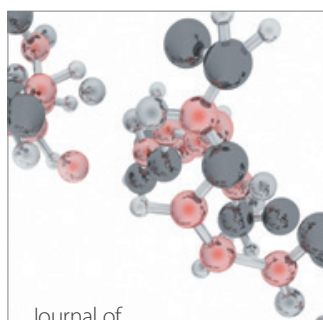

Analytical Methods

in Chemistry

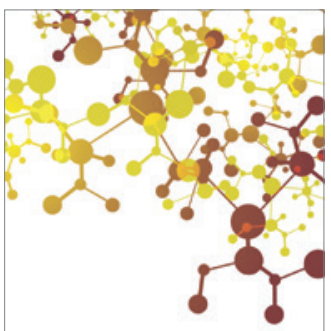

Journal of

Applied Chemistry

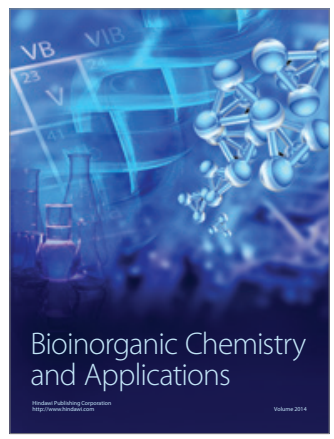

Inorganic Chemistry
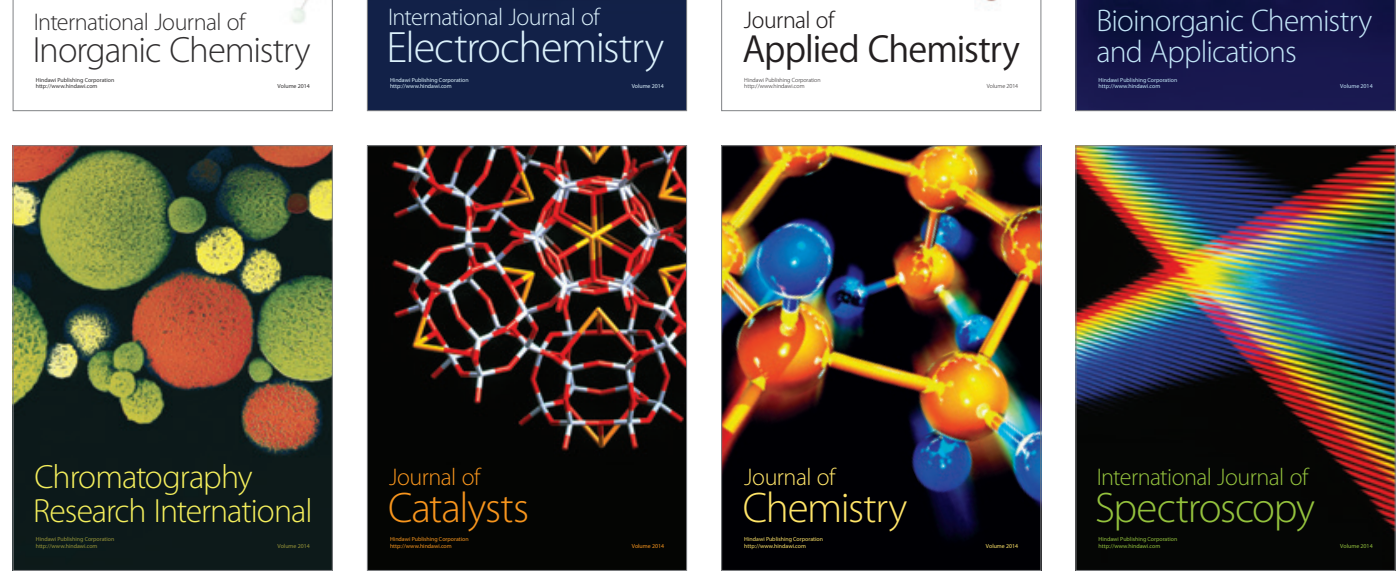